\title{
Basic operations for fuzzy multisets
}

\author{
A. Riesgo $^{\mathrm{a}}$, P. Alonso ${ }^{\mathrm{a}}$, I. Díaz ${ }^{\mathrm{b}}, \mathrm{S}$. Montes $^{\mathrm{c}}$ \\ ${ }^{a}$ Department of Mathematics, University of Oviedo, Spain \\ ${ }^{b}$ Department of Informatics, University of Oviedo, Spain \\ ${ }^{c}$ Department of Statistics and O.R., University of Oviedo, Spain
}

\begin{abstract}
In their original and ordinary formulation, fuzzy sets associate each element in a reference set with one number, the membership value, in the real unit interval $[0,1]$. Among the various existing generalisations of the concept, we find fuzzy multisets. In this case, membership values are multisets in $[0,1]$ rather than single values. Mathematically, they can be also seen as a generalisation of the hesitant fuzzy sets, but in this general environment, the information about repetition is not lost, so that, the opinions given by the experts are better managed. Thus, we focus our study on fuzzy multisets and their basic operations: complement, union and intersection. Moreover, we show how the hesitant operations can be worked out from an extension of the fuzzy multiset operations and investigate the important role that the concepts of order and sorting sequences play in the basic difference between these two related approaches.
\end{abstract}

Keywords: Fuzzy multiset; Hesitant fuzzy set; Complement; Aggregate union; Aggregate intersection.

\section{Introduction}

The concept of a fuzzy set was originally introduced by Lotfi A. Zadeh as an extension of classical set theory [15]. Fuzzy sets aim to account for real-life situations where there is either limited knowledge or some sort of implicit ambiguity about whether an element should be considered a member of a set. This extension from the classical (or crisp) sets to the fuzzy ones is accomplished by replacing the Boolean characteristic function of a set, which maps each element of the universal set to either 0 (non-member) or 1 (member), with a more sophisticated membership function that maps elements into the real interval $[0,1]$.

Fuzzy sets therefore model uncertainty about the membership of an element to a set, but it may appear paradoxical that the membership value itself should be one precise real number. In fact, generalisations of the idea where the membership value is replaced by more sophisticated mathematical entities, such as intervals (interval-valued fuzzy sets [6]) or functions (type-2 fuzzy sets [6]), have been proposed and make up a good deal of the ongoing research about fuzzy sets. Fuzzy multisets and hesitant fuzzy sets are the two generalisations that we are going to discuss in this paper. They are peculiar among the fuzzy set extensions in that, rather than modelling a tolerance around a certain central value, they model the uncertainty that occurs when several alternative membership values can be chosen; for example, when the membership value can be obtained through a number of related criteria that lead to different membership values.

The membership values in hesitant fuzzy sets can be arbitrary subsets of $[0,1]$ in their general formulation, but we will restrict our analysis to the particular case when these subsets are finite. Such hesitant fuzzy sets are called typical hesitant fuzzy sets [11] and they are the ones that have attracted the most attention from researchers $([3,7,14])$.

In fuzzy multisets, the membership value is a multiset in the $[0,1]$ real interval. If we restrict our study to the finite case, then the typical hesitant fuzzy sets can equivalently be represented as fuzzy multisets, the

Email addresses: ariesgo@yahoo.com (A. Riesgo), palonso@uniovi.es (P. Alonso), sirene@uniovi.es (I. Díaz), montes@uniovi.es (S. Montes) 
difference being that the fuzzy multisets allow repetition of the individual membership values. The need to account for repeated membership values has been recognised in the literature about hesitant fuzzy sets and, in fact, multiset-based hesitant fuzzy sets were already mentioned in the original paper that introduced the hesitant fuzzy sets [11]. But despite the similarities, we cannot regard the typical hesitant fuzzy sets as a particular case of the fuzzy multisets and neither can we identify the fuzzy multisets with the multiset-based hesitant fuzzy sets because, as we will see, the definitions for the intersection and union are different in each theory. The goal of this paper is to establish the appropriate mathematical definitions for the main operations for fuzzy multisets and show how the hesitant theory definitions can be worked out from an extension of the fuzzy multiset definitions.

This paper is organised as follows: Section 2 introduces the fundamental results of the existing theories of fuzzy sets, hesitant fuzzy sets and fuzzy multisets. Section 3 introduces new more general definitions for the intersection and union of fuzzy multisets. Section 4 proves that these new definitions for the intersection and union of fuzzy multisets can be identified with the definitions used for hesitant fuzzy sets and a bijection between the hesitant fuzzy sets and a subfamily of the fuzzy multisets is established. Finally, in section 5 we sum up the main conclusions of our research.

\section{Preliminary concepts}

In this section, we assume that there is always an axiomatic reference set or universe (also called the universe of discourse), which we will denote by $X$, and that fuzzy sets are defined through a membership function whose precise definition depends on the particular style of fuzzy sets we are dealing with.

\subsection{Fuzzy sets}

Definition 2.1. [15] Let $X$ be the universe. An fuzzy set $A$ is characterised by a function $A: X \rightarrow[0,1]$.

As we have mentioned above, we can see this function as a generalisation of the characteristic (or "indicator") function for a crisp set. Thus, in the particular case where $A(x)$ is always 0 or 1 , it can be regarded as a characteristic function into $\{0,1\}$. The values in the image set of $A$ are usually called the membership values of the fuzzy set and the family of all the fuzzy sets over $X$ is called the fuzzy power set over $X$ and is denoted by $\mathscr{F}(X)$.

Before introducing the generalisations of the fuzzy sets, we will first define the common operations for the fuzzy sets such as the complement, the intersection and the union. These definitions provide the backdrop against which the more sophisticated definitions for the hesitant fuzzy sets and fuzzy multisets can be compared.

Definition 2.2. [15] Let $X$ be the universe and let $A$ and $B$ be two fuzzy set over $X$.

- The standard complement $A^{c}$ is defined as the fuzzy set over $X$ such that

$$
A^{c}(x)=1-A(x), \quad \forall x \in X .
$$

- The standard intersection $A \cap B$ is defined as the fuzzy set over $X$ such that

$$
(A \cap B)(x)=\min \{A(x), B(x)\}, \quad \forall x \in X .
$$

- The standard union $A \cup B$ is defined as the fuzzy set over $X$ such that

$$
(A \cup B)(x)=\max \{A(x), B(x)\}, \quad \forall x \in X .
$$

It is straightforward to check that the above definitions reduce to the well-known classical set operations for crisp sets. In the available introductions to fuzzy set theory, more general definitions of these operations can be found (see, for instance [6]). This leads to a family of possible fuzzy complements, possible fuzzy intersections (defined in terms of $[0,1]^{2} \rightarrow[0,1]$ functions called $t$-norms) and possible fuzzy unions (defined in terms of similar functions called $t$-conorms). The standard ones just happen to be the best-behaved cases of the general operations. 


\subsection{Hesitant fuzzy sets}

A generalisation of the fuzzy sets was proposed by V. Torra in 2010 under the name hesitant fuzzy set theory [11], which builds on an idea that had already been put forward by I. Grattan-Guinness more than three decades earlier [5]. The hesitant theoretical framework broadens the range of the membership functions to encompass any subset of the $[0,1]$ interval.

The hesitant fuzzy sets have been widely studied in many papers published over the last few years (see, among many others, $[1,2,4,9,12]$ ). A comprehensive introduction can be found in a recent book by $\mathrm{Xu}$ [13]. In this section, we are going to sum up their formal definition and their main properties; these results are well-known and we will simply reproduce them here for completeness.

Definition 2.3. [11] Let $X$ be the universe. A hesitant fuzzy set $\tilde{A}$ over $X$ is characterised by a function $\tilde{A}: X \rightarrow \mathcal{P}([0,1])$, where $\mathcal{P}([0,1])$ is the family of all the subsets of the real closed interval $[0,1]$.

The family of all the hesitant fuzzy sets over $X$ is called the hesitant fuzzy power set over $X$ and is denoted by $\mathscr{F} \mathscr{H}(X)$.

Given any hesitant fuzzy set $\tilde{A}$ over a universe $X$, the subsets of $[0,1]$ that form the image of $\tilde{A}$ are commonly referred to as hesitant elements [13]. We will restrict our analysis to those hesitant fuzzy sets such that their hesitant elements are finite, which are called typical hesitant fuzzy sets [11].

Now we are going to recall the definitions of complement, intersection and union operations for the hesitant fuzzy sets.

Definition 2.4. [11] Let $X$ be the universe and let $\tilde{A}$ and $\tilde{B} \in \mathscr{F} \mathscr{H}(X)$ be two hesitant fuzzy sets. Then:

- The complement of $\tilde{A}$ is the hesitant fuzzy set $\tilde{A}^{c}$ defined by a function $\tilde{A}^{c}: X \rightarrow \mathcal{P}([0,1])$ such that

$$
\tilde{A}^{c}(x)=\bigcup_{\gamma \in \tilde{A}(x)}\{1-\gamma\}, \quad \forall x \in X
$$

- The intersection of $\tilde{A}$ and $\tilde{B}$ is the hesitant fuzzy set $\tilde{A} \cap \tilde{B}$ defined by a function $(\tilde{A} \cap \tilde{B}): X \rightarrow \mathcal{P}([0,1])$ such that

$$
(\tilde{A} \cap \tilde{B})(x)=\{\alpha \in \tilde{A}(x) \cup \tilde{B}(x) \mid \alpha \leq \min \{\max \{\tilde{A}(x)\}, \max \{\tilde{B}(x)\}\}\}, \quad \forall x \in X .
$$

- The union of $\tilde{A}$ and $\tilde{B}$ is the hesitant fuzzy set $\tilde{A} \cup \tilde{B}$ defined by a function $(\tilde{A} \cup \tilde{B}): X \rightarrow \mathcal{P}([0,1])$ such that

$$
(\tilde{A} \cup \tilde{B})(x)=\{\alpha \in \tilde{A}(x) \cup \tilde{B}(x) \mid \alpha \geq \max \{\min \{\tilde{A}(x)\}, \min \{\tilde{B}(x)\}\}\}, \quad \forall x \in X .
$$

Unlike the definition for the complement, these definitions for the intersection and the union are not totally intuitive. But they are consistent with the requirement that they reduce to the ordinary definitions with single-valued hesitant elements and, when hesitant fuzzy sets are regarded as a form of type-2 fuzzy sets (in their general form, without any convexity assumptions), can also be shown to be consistent with the type-2 fuzzy set definitions that were established by Mizumoto and Tanaka [8] in 1976. In this paper, we will provide an alternative justification for these definitions.

It is easy to prove the intuitive result that the fuzzy sets are a particular case of the hesitant fuzzy sets. This can be established formally by defining a bijection between the fuzzy power set over a universe $X$ and a convenient subset of the hesitant fuzzy power set over $X$. Thus, given any fuzzy set $A \in \mathscr{F}(X)$, we can define the hesitant element $\phi_{\mathrm{FH}}(A)$ as $\phi_{\mathrm{FH}}(A)(x)=\{A(x)\}$. It is obvious that $\phi_{\mathrm{FH}}$ is a bijection. With this natural identification between the fuzzy sets and the hesitant fuzzy sets with hesitant element cardinality 1 , we can also prove that the operations of complement, intersection and union are compatible with the bijection. 
Example 2.5. If we have a single-element universe $X=\{x\}$, we can define two trivial hesitant fuzzy sets $\tilde{A}(x)=\{0.1\}$ and $\tilde{B}(x)=\{0.2\}$. Their complements are $\tilde{A}^{c}(x)=\{0.9\}$ and $\tilde{B}^{c}(x)=\{0.8\}$ and their intersection and union $\tilde{A} \cap \tilde{B}(x)=\{0.1\}$ and $\tilde{A} \cup \tilde{B}(x)=\{0.2\}$. These results are exactly the same as what would be expected with two fuzzy sets defined by $A(x)=0.1$ and $B(x)=0.2$. In the non-trivial case, we can have two hesitant fuzzy sets like $\tilde{C}(x)=\{0.10,0.11\}$ and $\tilde{D}(x)=\{0.20,0.21\}$ and we would have $\tilde{C}^{c}(x)=\{0.89,0.90\}, \tilde{D}^{c}(x)=\{0.79,0.80\}, \tilde{C} \cap \tilde{D}(x)=\{0.10,0.11\}$ and $\tilde{C} \cup \tilde{D}(x)=\{0.20,0.21\}$. This simple example reveals the intuition behind the hesitant operations.

\subsection{Fuzzy multisets}

As we mentioned in the introduction, the values that make up a hesitant element in a hesitant fuzzy set are typically the result of applying several criteria on membership. In a common use case, it is assumed that there are a number of "experts" or "decision-makers" for a hesitant fuzzy set who produce a membership value for each element in the universe. A problem with the hesitant fuzzy sets in the experts' model is that the information about repetition is lost. For example, if there are five experts and four of them assign a membership value of 0.1 to an element whereas the fifth expert assigns the value 0.2 , the hesitant element will be $\{0.1,0.2\}$, regardless of the fact that 0.1 was four times more popular among the experts. This information loss can be avoided by using fuzzy multisets [7] (also called fuzzy bags [14]), which we are going to discuss now.

But in order to state a formal definition, we will first need to review the multisets in a crisp sense $[7,10]$. To avoid confusion with the similar fuzzy concepts, we will denote in general the universe of discourse by $U$ rather than $X$ in the definitions and results related to the crisp multisets. Along this paper, $U$ will really be the closed interval $[0,1]$.

Definition 2.6. Let $U$ be a universe. A (crisp) multiset $M$ over the universe $U$ is characterised by a

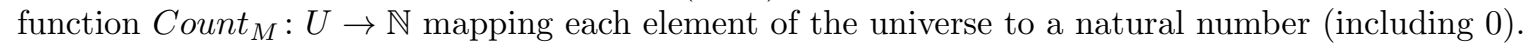

The family of all the crisp multisets over $U$ is called the power multiset of $U$ and is denoted by $\mathbb{N}^{U}$.

As in the case of the fuzzy sets and their membership functions, although the concepts of "multiset" and "count function" are formally equivalent, it is common to refer to a multiset $M$ and its count function Count $_{M}$ as distinct mathematical entities.

The concept of cardinality can be extended to the multisets in a natural way:

Definition 2.7. Let $M$ be a multiset over a universe $U$. The cardinality of $M$ is the natural number defined as

$$
|M|:=\sum_{u \in U} \text { Count }_{M}(u)
$$

In this paper, we restrict our analysis to the multisets with a finite cardinality, which we will call finite multisets.

As we would expect, those multisets having a count function with the image set restricted to $\{0,1\}$ can be identified with the ordinary sets. In that particular case, the count function becomes equivalent to the characteristic function of the set and the cardinality can be interpreted in the ordinary sense too. So the multisets behave as an extension of the ordinary concept of a set, and in this regard share some characteristics with the fuzzy sets.

In fact, the definitions of some common operations for multisets are quite similar to those of fuzzy sets. Here we are going to define the union, which we will use later.

Definition 2.8. Let $M, N \in \mathbb{N}^{U}$ be two finite multisets over a universe $U$. The union of $M$ and $N$ is the multiset $M \cup N$ defined by a function $\operatorname{Count}_{M \cup N}: U \rightarrow \mathbb{N}$ such that

$$
\operatorname{Count}_{M \cup N}(u)=\max \left\{\operatorname{Count}_{M}(u), \operatorname{Count}_{N}(u)\right\}, \quad \forall u \in U .
$$


The definition of intersection, which we will not use in this paper, is completely analogous, with min replacing max.

Now that we have defined the crisp multisets, together with their most fundamental results, we can introduce the fuzzy multisets as follows,

Definition 2.9. [7] Let $X$ be the universe. A fuzzy multiset $\hat{A}$ over $X$ is characterised by a function $\hat{A}: X \rightarrow \mathbb{N}^{[0,1]}$. The family of all the fuzzy multisets over $X$ is called the fuzzy power multiset over $X$ and is denoted by $\mathscr{F} \mathscr{M}(X)$.

Example 2.10. Say we have a single-element universe $X=\{x\}$. We can define a fuzzy multiset $\hat{A}$ as $\hat{A}(x)=\langle 0.1,0.2,0.2\rangle$ in angular-bracket notation. Or in other words, using Definition 2.9 , the element $x$

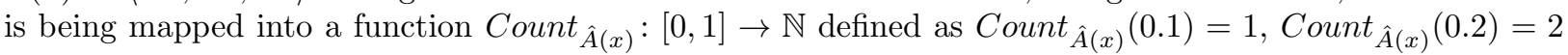
and Count $_{\hat{A}(x)}(t)=0$ for any $t \neq 0.1$ and $t \neq 0.2$. This function Count $_{\hat{A}(x)}$ characterises a crisp multiset for any $x$ in $X$.

Now we are going to define the complement, intersection and union operations for the fuzzy multisets. The complement for the fuzzy multisets is quite intuitive.

Definition 2.11. [7] Let $X$ be a universe and let $\hat{A} \in \mathscr{F} \mathscr{M}(X)$ be a fuzzy multiset. The complement of $\hat{A}$ is the fuzzy multiset $\hat{A}^{c}$ defined by the following count function:

$$
\text { Count }_{\hat{A}^{c}(x)}(t)=\text { Count }_{\hat{A}(x)}(1-t), \quad \forall x \in X, \quad \forall t \in[0,1] .
$$

Example 2.12. If we have a two-element universe $X=\{x, y\}$, then a fuzzy multiset $\hat{A}$ with $\hat{A}(x)=\langle 0.3\rangle$ and $\hat{A}(y)=\langle 0.5,0.8,0.8\rangle$ has the complement

$$
\operatorname{Count}_{\hat{A}^{c}(x)}(t)=\left\{\begin{array}{cc}
1, & \text { if } t=0.7, \\
0, & \text { otherwise, }
\end{array} \quad \text { Count }_{\hat{A}^{c}(y)}(t)=\left\{\begin{array}{cc}
1, & \text { if } t=0.5, \\
2, & \text { if } t=0.2, \\
0, & \text { otherwise }
\end{array}\right.\right.
$$

that is, $\hat{A}^{c}(x)=\langle 0.7\rangle$ and $\hat{A}(y)=\langle 0.5,0.2,0.2\rangle$.

The definitions of intersection and union have been more problematic in the literature. In his original formulation of fuzzy bags [14], Yager proposed a definition that did not work as a general case of the fuzzy set definition. A better definition was proposed by Miyamoto [7]. In Miyamoto's approach, the definition of these binary operations between fuzzy multisets is made simpler by working with membership values that have the same cardinality. We will develop this approach formally by first defining subfamilies of the fuzzy multisets where the cardinality is fixed to a constant finite value for each member of the universe $X$.

Definition 2.13. Let $X$ be a universe. Given a function $m: X \rightarrow \mathbb{N}$, an $m$-regular fuzzy multiset $\hat{A}$ over the universe $X$ is a fuzzy multiset such that, for each element of the universe $x \in X$, the membership multiset of $\hat{A}$ has a cardinality $|\hat{A}(x)|=m(x)$. We will call the $m$ function a cardinality map.

The family of all the $m$-regular fuzzy multisets over $X$ is called the $m$-regular fuzzy power multiset and is denoted by $\mathscr{F} \mathscr{M}^{m}(X)$.

Example 2.14. If we have a two-element universe $X=\{x, y\}$, then a fuzzy multiset $\hat{A}$ with $\hat{A}(x)=$ $\langle 0.2,0.3\rangle$ and $\hat{A}(y)=\langle 0.5,0.8,0.9\rangle$ is an example of an $m$-regular fuzzy multiset with $m(x)=2$ and $m(y)=3$. 
The importance of this concept lies in the fact that if we have a second fuzzy multiset $\hat{B}$, we can easily define binary operations between $\hat{A}$ and $\hat{B}$, like the intersection and the union, by operating on their membership values one by one, but we can only do that if $\hat{B}$ is also $m$-regular. So, restricting the operations to the regular fuzzy multisets provides a convenient first step in definitions.

Following Miyamoto, in order to define the binary operations it is also convenient to be able to arrange the elements in a membership multiset into a sequence. For this we will need some formal definitions for the crisp multisets.

Definition 2.15. Let $U$ be a universe. For any finite multiset $M$ over $U$, an ordered sequence of $M$ is an ordered $|M|$-tuple $\left(a_{1}, \ldots, a_{|M|}\right) \in U^{|M|}$ such that for each $u \in U$ with $\operatorname{Count}_{M}(u)>0$ there are $\operatorname{Count}_{M}(u)$ coordinates equalling $u$; i.e. $\left|\left\{i \in\{1, \ldots,|M|\} \mid a_{i}=u\right\}\right|=$ Count $_{M}(u)$. Thus, an ordering strategy is any function $s: \mathbb{N}_{\text {finite }}^{U} \rightarrow \mathscr{O} \mathscr{S}(U)$ that maps a finite multiset to an ordered sequence, where $\mathscr{O} \mathscr{S}(U)$ denotes the family of all the ordered sequences for any multiset over $U$.

Really, an ordering strategy maps any finite multiset to a tuple with the same cardinality as the multiset, obtaining just as a permutation of the values of the multiset. The usual ordering strategy consists in sorting in either ascending or descending order, which can be done when the universe $U$ is totally ordered. Thus, the ascending sort of a multiset $M$ is the ordering strategy that maps $M$ to an ordered sequence $s^{\uparrow}(M)$ such that if $i, j \in\{1, \ldots,|M|\}$ with $i<j$, then $\left(s^{\uparrow}(M)\right)_{i} \leq\left(s^{\uparrow}(M)\right)_{j}$. Similarly, the descending sort is the ordering strategy $s_{\downarrow}$ such that if $i, j \in\{1, \ldots,|M|\}$ with $i<j$, then $\left(s_{\downarrow}(M)\right)_{i} \geq\left(s_{\downarrow}(M)\right)_{j}$.

Example 2.16. If the universe is the real unit interval $[0,1]$ and we have a multiset $M=\langle 0.3,0.2,0.2,0.5\rangle$, an ordered sequence $a$ is simply an arrangement of these four values in a specific order, for example $a=$ $(0.2,0.2,0.3,0.5)$, and the function $s$ that maps $M$ to $a$ is the ordering strategy. In this case we are sorting the elements in ascending order, so $s=s^{\uparrow}$. The descending sort $s_{\downarrow}$ would yield $(0.5,0.3,0.2,0.2)$ but any arbitrary arrangement like $(0.5,0.2,0.3,0.2)$ is a valid example of an ordered sequence.

The definitions of intersection and union due to Miyamoto are based on operating in a coordinatewise fashion on the ordered sequences that result from picking the descending sort $s_{\downarrow}$ as the ordering strategy.

Definition 2.17. Let $X$ be a universe and let $m: X \rightarrow \mathbb{N}$ be a cardinality map. Given two $m$-regular fuzzy multisets $\hat{A}$ and $\hat{B}$, its $m$-regular intersection $\hat{A} \cap \hat{B}$ is the $m$-regular fuzzy multiset such that

$$
\operatorname{Count}_{(\hat{A} \cap \hat{B})(x)}(t)=\left|\left\{i \mid 1 \leq i \leq m(x),\left(\mu_{\hat{A} \cap \hat{B}}(x)\right)_{i}=t\right\}\right|
$$

where

$$
\left(\mu_{\hat{A} \cap \hat{B}}(x)\right)_{i}:=\min _{i \in\{1, \ldots, m(x)\}}\left\{\left(s_{\downarrow}(\hat{A}(x))\right)_{i},\left(s_{\downarrow}(\hat{B}(x))\right)_{i}\right\} .
$$

Similarly, we can define the union as

Definition 2.18. Let $X$ be a universe and let $m: X \rightarrow \mathbb{N}$ be a cardinality map. Given two $m$-regular fuzzy multisets $\hat{A}$ and $\hat{B}$, its $m$-regular union $\hat{A} \cup \hat{B}$ is the $m$-regular fuzzy multiset such that

$$
\operatorname{Count}_{(\hat{A} \cup \hat{B})(x)}(t)=\left|\left\{i \mid 1 \leq i \leq m(x),\left(\mu_{\hat{A} \cup \hat{B}}(x)\right)_{i}=t\right\}\right|
$$

where

$$
\left(\mu_{\hat{A} \cup \hat{B}}(x)\right)_{i}:=\max _{i \in\{1, \ldots, m(x)\}}\left\{\left(s_{\downarrow}(\hat{A}(x))\right)_{i},\left(s_{\downarrow}(\hat{B}(x))\right)_{i}\right\} .
$$

Note that choosing $s_{\downarrow}$ or $s_{\uparrow}$ in the above definition is just a matter of convention as it can easily be proved that both ordering strategies yield the same numeric values.

When the two fuzzy multisets $\hat{A}$ and $\hat{B}$ have different cardinalities for an element $x \in X$, we will be able to apply the previous definitions if we perform an additional step, which we will call regularisation, that equalises the cardinalities to the maximum of the two. This operation can be formally defined as follows, 
Definition 2.19. Let $X$ be a universe and let $\hat{A} \in \mathscr{F} \mathscr{M}(X)$ be a fuzzy multiset. Given a cardinality map $m: X \rightarrow \mathbb{N}$ such that $|\hat{A}(x)| \leq m(x)$ for all $x \in X$, a regularisation of $\hat{A}$ to cardinality $m$ is a fuzzy multiset $\hat{A}^{r}$ such that:

$$
\begin{aligned}
& \left|\hat{A}^{r}(x)\right|=m(x), \quad \forall x \in X, \\
& \operatorname{Count}_{\hat{A}^{r}(x)}(t)=0, \quad \text { if } \operatorname{Count}_{\hat{A}(x)}(t)=0, \\
& \operatorname{Count}_{\hat{A}^{r}(x)}(t) \geq \text { Count }_{\hat{A}(x)}(t), \quad \text { if } \operatorname{Count}_{\hat{A}(x)}(t)>0 .
\end{aligned}
$$

A similar definition was considered in [7], although it was not formally defined. It is straightforward to check that in the trivial case when $|\hat{A}(x)|=m(x)$ for all $x \in X, \hat{A}^{r}(x)=\hat{A}(x)$.

Example 2.20. If we have a fuzzy multiset $\hat{A}$ over a single-element universe $X=\{x\}$ and $\hat{A}(x)=$ $\langle 0.1,0.2,0.2\rangle$, a regularisation to cardinality 5 would consist in incrementing the count of some of the members in order to reach that cardinality. $\hat{A}^{r 1}=\langle 0.1,0.1,0.1,0.2,0.2\rangle$ and $\hat{A}^{r 2}=\langle 0.1,0.2,0.2,0.2,0.2\rangle$ would be two such regularisations. Thus, we can see in this example that the regularisation is not unique.

The assignment of a particular regularisation to a fuzzy multiset for larger cardinalities will be referred to as the regularisation strategy. The two most common regularisation strategies consist in increasing either the count of the maximum membership value or that of the minimum. Following a common convention in the hesitant literature [13], we will refer to these two approaches as optimistic and pessimistic, respectively.

With this additional concept of regularisation we can now define the intersection and union of any arbitrary pair of fuzzy multisets regardless of their respective cardinalities. But we need an additional definition first.

Definition 2.21. Let $X$ be a universe and let $\hat{A}, \hat{B} \in \mathscr{F} \mathscr{M}(X)$ be two fuzzy multisets. Their maximum common cardinality map is the cardinality map $m_{\hat{A} \hat{B}}$ defined as

$$
m_{\hat{A} \hat{B}}(x)=\max _{x \in X}\{|\hat{A}(x)|,|\hat{B}(x)|\} .
$$

In general, by regularising to their maximum common cardinality map, we arrive at a definition of intersection for any pair of fuzzy multisets.

Definition 2.22. Let $X$ be a universe and let $\hat{A}, \hat{B} \in \mathscr{F} \mathscr{M}(X)$ be two fuzzy multisets. Given a regularisation strategy that assigns a pair of fuzzy multisets $\hat{A}^{r}, \hat{B}^{r} \in \mathscr{F} \mathscr{M}(X)$ regularised to their maximum common cardinality map $m_{\hat{A} \hat{B}}$. Then:

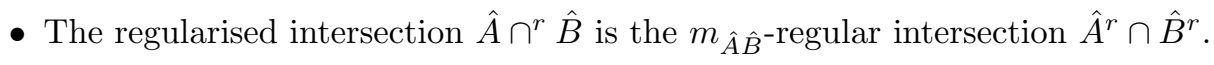

- The regularised union $\hat{A} \cup \cup^{r} \hat{B}$ is the $m_{\hat{A} \hat{B}^{-r e g u l a r}}$ union $\hat{A}^{r} \cup \hat{B}^{r}$.

Example 2.23. If we have a two-element universe $X=\{x, y\}$ and an fuzzy multiset $\hat{A}$ such that $\hat{A}(x)=$ $\langle 0.2,0.3\rangle$ and $\hat{A}(y)=\langle 0.5,0.6,0.6\rangle$ and another fuzzy multiset $\hat{B}$ such that $\hat{B}(x)=\langle 0.6,0.6\rangle$ and $\hat{B}(y)=$ $\langle 0.1,0.2,0.3\rangle$, as they are both $\{2,3\}$-regular, they are trivially regularised and their intersection is $\hat{A} \cap \hat{B}(x)$ $=\langle 0.2,0.3\rangle$ and $\hat{A} \cap \hat{B}(y)=\langle 0.1,0.2,0.3\rangle$. But if we have an fuzzy multiset $\hat{C}$ such that $\hat{C}(x)=\langle 0.2\rangle$ and $\hat{C}(y)=\langle 0.2,0.8\rangle$, we will need to regularise $\hat{C}$ to the larger cardinalities of $\hat{A}$. With an optimistic regularisation, we get $\hat{C}^{r}$ defined as $\hat{C}^{r}(x)=\langle 0.2,0.2\rangle$ and $\hat{C}^{r}(y)=\langle 0.2,0.8,0.8\rangle$ and then we can perform the intersection $\hat{A} \cap^{r+} \hat{C}(x)=\langle 0.2,0.2\rangle$ and $\hat{A} \cap^{r+} \hat{C}(y)=\langle 0.1,0.2,0.3\rangle$.

Just as we did in the previous subsection, 2.2 , it can easily be proved that, given a universe $X$, there is a bijection $\phi_{F M}$ between the family of the 1 -regular fuzzy sets $\mathscr{F} \mathscr{M}^{1}(X)$ (where 1 stands for the unit cardinality map $1(x)=1$ for all $x \in X)$ and the fuzzy power set $\mathscr{F}(X)$ and that the standard complement, 
standard intersection and standard union, defined on $\mathscr{F}(X)$, and the 1 -regular complement, intersection and union, defined on $\mathscr{F} \mathscr{M}^{1}(X)$, respectively commute with $\phi_{F M}$. This means that the fuzzy multisets, like the hesitant fuzzy sets, are an extension of the fuzzy sets.

But what, if any, is the connection between the fuzzy multisets and the hesitant fuzzy sets? Can we establish a similar bijection with compatible operations between at least some subsets of either family? As we show in the next example, the situation is tricky because of the different behaviours of the intersection and union operations. In the next section we are going to define new intersection and union operations that make this possible.

Example 2.24. In a similar example to Example 2.5, if we have a single-element universe $X=\{x\}$, we can define two trivial fuzzy multisets $\hat{A}(x)=\langle 0.1\rangle$ and $\hat{B}(x)=\langle 0.2\rangle$. Their complements are $\hat{A}^{c}(x)=\langle 0.9\rangle$ and $\hat{B}^{c}(x)=\langle 0.8\rangle$ and their 1 -regular intersection and union are $\hat{A} \cap \hat{B}(x)=\langle 0.1\rangle$ and $\hat{A} \cup \hat{B}(x)=\langle 0.2\rangle$. As in the hesitant fuzzy set example, the results contain the same numeric values as with the two fuzzy sets defined by $A(x)=0.1$ and $B(x)=0.2$.

With non-trivial fuzzy multisets, we can also find many cases that yield the same numeric values as the similar hesitant fuzzy sets. For example, if we have two fuzzy multisets like $\hat{C}(x)=\langle 0.10,0.11\rangle$ and $\hat{D}(x)=\langle 0.20,0.21\rangle$, we get $\hat{C}^{c}(x)=\langle 0.89,0.90\rangle, \hat{D}^{c}(x)=\langle 0.79,0.80\rangle, \hat{C} \cap \hat{D}(x)=\langle 0.10,0.11\rangle$ and $\hat{C} \cup \hat{D}(x)=$ $\langle 0.20,0.21\rangle$. This shows that the intuition behind the two approaches is similar.

But we do find situations where each theory leads to different results for the intersection and union. This happens when there are overlapping ranges of values in the two sets. For example, if we have the two fuzzy multisets $\hat{E}(x)=\langle 0.1,0.4\rangle$ and $\hat{F}(x)=\langle 0.2,0.3\rangle$, we get $\hat{E} \cap \hat{F}(x)=\langle 0.1,0.3\rangle$ and $\hat{E} \cup \hat{F}(x)=\langle 0.2,0.4\rangle$. But if we have the two analogous hesitant fuzzy sets $\tilde{E}(x)=\{0.1,0.4\}$ and $\tilde{F}(x)=\{0.2,0.3\}$, we get $\tilde{E} \cap \tilde{F}(x)=\{0.1,0.2,0.3\}$ and $\tilde{E} \cup \tilde{F}(x)=\{0.2,0.3,0.4\}$. We see that the hesitant intersection and union can change the cardinality of the hesitant elements when the two sets have overlapping ranges of values, a phenomenon that does not occur with the fuzzy multisets.

\section{New definitions of intersection and union for fuzzy multisets}

In Miyamoto's definitions of the intersection and union, sorting plays an essential part but it should be noted that the approach based on the pairwise minima and maxima can be applied to any other ordering strategy. In fact, in the experts' model where a number of experts produce the membership values in the multiset, there may be an underlying natural order (based on pairing those values coming from the same expert) that is disrupted by sorting.

This leads to a first idea that we can craft a more general definition of what we have called the $m$-regular intersection (Definition 2.17) and union (Definition 2.18) by turning the ordering strategies into parameters.

Definition 3.1. Let $X$ be a universe and let $m: X \rightarrow \mathbb{N}$ be a cardinality map. Given two $m$-regular fuzzy multisets $\hat{A}$ and $\hat{B}$ and two ordering strategies $s_{A}$ and $s_{B}$, for each element $x \in X$, a new sequence $\mu_{\hat{A}_{\left(s_{A}, s_{B}\right)} \hat{B}}(x)$ can be built with the pairwise minima as follows

$$
\left(\mu_{\hat{A} \cap_{\left(s_{A}, s_{B}\right)} \hat{B}}(x)\right)_{i}:=\min _{i \in\{1, \ldots, m(x)\}}\left\{\left(s_{A}(\hat{A}(x))\right)_{i},\left(s_{B}(\hat{B}(x))\right)_{i}\right\} .
$$

The $m$-regular $\left(s_{A}, s_{B}\right)$-ordered intersection $\hat{A} \cap_{\left(s_{A}, s_{B}\right)} \hat{B}$ is the $m$-regular fuzzy multiset defined by

$$
\operatorname{Count}_{\left(\hat{A} \cap_{\left(s_{A}, s_{B}\right)} \hat{B}\right)(x)}(t)=\left|\left\{i \mid 1 \leq i \leq m(x),\left(\mu_{\hat{A} \cap_{\left(s_{A}, s_{B}\right)} \hat{B}}(x)\right)_{i}=t\right\}\right| .
$$

Similarly, we can obtain a definition the union.

Definition 3.2. Let $X$ be a universe and let $m: X \rightarrow \mathbb{N}$ be a cardinality map. Given two $m$-regular fuzzy multisets $\hat{A}$ and $\hat{B}$ and two ordering strategies $s_{A}$ and $s_{B}$, for each element $x \in X$, a new sequence $\mu_{\hat{A} \cup_{\left(s_{A}, s_{B}\right)} \hat{B}}(x)$ can be built with the pairwise maxima as follows

$$
\left(\mu_{\hat{A} \cup_{\left(s_{A}, s_{B}\right)} \hat{B}}(x)\right)_{i}:=\max _{i \in\{1, \ldots, m(x)\}}\left\{\left(s_{A}(\hat{A}(x))\right)_{i},\left(s_{B}(\hat{B}(x))\right)_{i}\right\} .
$$


The $m$-regular $\left(s_{A}, s_{B}\right)$-ordered union $\hat{A} \cup_{\left(s_{A}, s_{B}\right)} \hat{B}$ is the $m$-regular fuzzy multiset defined by

$$
\operatorname{Count}_{\left(\hat{A} \cup_{\left(s_{A}, s_{B}\right)} \hat{B}\right)(x)}(t)=\left|\left\{i \mid 1 \leq i \leq m(x),\left(\mu_{\hat{A} \cup_{\left(s_{A}, s_{B}\right)} \hat{B}}(x)\right)_{i}=t\right\}\right| .
$$

And with these definitions that allow for mixed ordering, we can define new versions of the intersection and union for non-regular pairs of fuzzy multisets, analogous to Definition 2.22.

Definition 3.3. Let $X$ be a universe and let $\hat{A}, \hat{B} \in \mathscr{F} \mathscr{M}(X)$ be two fuzzy multisets. Given a regularisation strategy that assigns a pair of fuzzy multisets $\hat{A}^{r}, \hat{B}^{r} \in \mathscr{F} \mathscr{M}(X)$ regularised to their maximum common cardinality map $m_{\hat{A} \hat{B}}$ and two ordering strategies $s_{A}$ and $s_{B}$. Then:

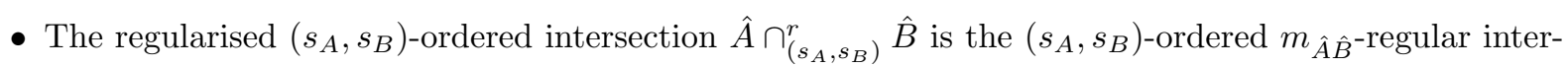
section $\hat{A}^{r} \cap_{\left(s_{A}, s_{B}\right)} \hat{B}^{r}$.

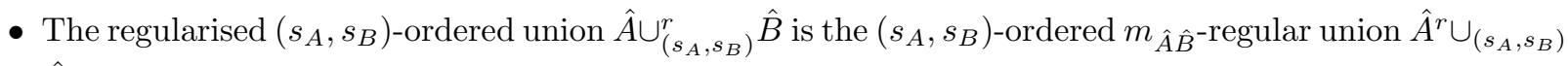
$\hat{B}^{r}$.

The previous definitions allow us to choose arbitrary and different ordering strategies for each fuzzy multiset in a binary operation. By taking the multiset union of all the combinations, we can define what we will call the aggregate intersection and union of two fuzzy multisets, which don't privilege any particular ordering.

Definition 3.4. Let $X$ be a universe and let $\hat{A}, \hat{B} \in \mathscr{F} \mathscr{M}(X)$ be two fuzzy multisets. The aggregate intersection of $\hat{A}$ and $\hat{B}$ is a fuzzy multiset $\hat{A} \cap^{a} \hat{B}$ such that for any element $x \in X, \hat{A} \cap^{a} \hat{B}(x)$ is the union, in the crisp multiset sense, of the regularised $\left(s_{A}, s_{B}\right)$-ordered intersections for all the possible pairs of ordering strategies $\left(s_{A}, s_{B}\right)$, that is,

$$
\hat{A} \cap^{a} \hat{B}(x)=\bigcup_{\substack{s_{\hat{A}} \in \mathcal{O S}\left(\hat{A}^{r}\right) \\ s_{\hat{B}} \in \mathcal{O S}\left(\hat{B}^{r}\right)}} \hat{A} \cap_{\left(s_{A}, s_{B}\right)}^{r} \hat{B}(x), \quad \forall x \in X .
$$

Definition 3.5. Let $X$ be a universe and let $\hat{A}, \hat{B} \in \mathscr{F} \mathscr{M}(X)$ be two fuzzy multisets. The aggregate union of $\hat{A}$ and $\hat{B}$ is a fuzzy multiset $\hat{A} \cup^{a} \hat{B}$ such that for any element $x \in X, \hat{A} \cup^{a} \hat{B}(x)$ is the union, in the crisp multiset sense, of the regularised $\left(s_{A}, s_{B}\right)$-ordered unions for all the possible pairs of ordering strategies $\left(s_{A}, s_{B}\right)$, that is,

$$
\hat{A} \cup^{a} \hat{B}(x)=\bigcup_{\substack{s_{\hat{A}} \in \mathcal{O} \mathcal{S}\left(\hat{A}^{r}\right) \\ s_{\hat{B}} \in \mathcal{O S}\left(\hat{B}^{r}\right)}} \hat{A} \cup_{\left(s_{A}, s_{B}\right)}^{r} \hat{B}(x), \quad \forall x \in X .
$$

Example 3.6. In Example 2.24, we showed that for two fuzzy multisets $\hat{E}(x)=\langle 0.1,0.4\rangle$ and $\hat{F}(x)=$ $\langle 0.2,0.3\rangle$, the Miyamoto intersection and union are $\hat{E} \cap \hat{F}(x)=\langle 0.1,0.3\rangle$ and $\hat{E} \cup \hat{F}(x)=\langle 0.2,0.4\rangle$. In order to calculate their aggregate intersection and union, we need to first calculate the interesections and unions for all the possible ordering strategies. There are two possible ordering strategies for $\hat{E}$, resulting in the sequences $(0.1,0.4)$ and $(0.4,0.1)$, and two possible ordering strategies for $\hat{F}$, resulting in the sequences $(0.2,0.3)$ and $(0.3,0.2)$. This leads to the four sequences of pairwise minima, $(0.1,0.3),(0.1,0.2),(0.2,0.1)$, $(0.3,0.1)$, which result in two ordered intersections, using Definition 3.1, $\langle 0.1,0.3\rangle,\langle 0.1,0.2\rangle$; and to the four sequences of pairwise maxima, $(0.2,0.4),(0.3,0.4),(0.4,0.3),(0.4,0.2)$, which result in two ordered unions, using Definition $3.2,\langle 0.2,0.4\rangle,\langle 0.3,0.4\rangle$. By taking the union, in the crisp multiset sense, we get the aggregate intersection and union: $\hat{E} \cap^{a} \hat{F}(x)=\langle 0.1,0.2,0.3\rangle$ and $\hat{E} \cup^{a} \hat{F}(x)=\langle 0.2,0.3,0.4\rangle$. We have found the striking result that the numeric values match those of the hesitant fuzzy set intersection and union in the previous examples, a hint that the hesitant theory is equivalent to the fuzzy multiset theory when the aggregate operations are used, as we will prove in the next section. 


\section{The fuzzy multisets as an extension of the hesitant fuzzy sets}

We are now going to show that the fuzzy multisets are an extension of the hesitant fuzzy sets with the aggregate-form definitions. This is obviously similar to the way a multiset like $\langle 0.1,0.2\rangle$ can be identified with the set $\{0.1,0.2\}$. We will first establish how the crisp multisets can be related to the underlying crisp sets that result from disregarding the multiplicity information by means of an equivalence relation that equalises the non-zero multiplicities. And then we will use that multiset-based equivalence relation to establish a similar relation between the fuzzy multisets and the hesitant fuzzy sets.

We have mentioned how we can identify the crisp sets with those crisp multisets having a count value of just 0 and 1 . An alternative way of identifying the crisp subsets with a restricted version of the family of multisets is through an equivalence relation that does away with the information on repeated elements.

First, by disregarding the actual count value and only considering whether it is non-zero, we can relate a multiset to an underlying set.

Definition 4.1. Let $U$ be a universe and let $M \in \mathbb{N}^{U}$ be a multiset over $U$. The support of $M$ is the crisp set of $U, \operatorname{Supp}_{m}(M), \in \mathcal{P}(U)$ defined as

$$
\operatorname{Supp}_{m}(M):=\left\{u \in U \mid \operatorname{Count}_{M}(u)>0\right\} .
$$

And, armed with this definition, we can go on to define an equivalence relation as follows,

Definition 4.2. Let $U$ be a universe and let $M, N \in \mathbb{N}^{U}$ be two multisets over $U . M$ and $N$ are said to be repetition-equivalent, $M \sim_{m r} N$, if their supports are the same: $\operatorname{Supp}_{m}(M)=\operatorname{Supp}_{m}(N)$.

It is trivial to prove that $\sim_{m r}$ is an equivalence relation.

Example 4.3. In the universe of the natural numbers $\mathbb{N}$, we can define two multisets $M=\langle 1,2\rangle$ and $N=\langle 1,2,2,2\rangle$. Since these multisets only differ in the count value for an element that appears in both, they are repetition-equivalent, $M \sim_{m r} N$, and their support is the crisp subset $\{1,2\}$ in both cases.

Having established the required concepts for the crisp multisets, we can now define an underlying hesitant fuzzy set for any fuzzy multiset.

Definition 4.4. Let $X$ be a universe and let $\hat{A} \in \mathscr{F} \mathscr{M}(X)$ be a fuzzy multiset over $X$. The hesitant fuzzy set support of $\hat{A}$ is a hesitant fuzzy set $\operatorname{Supp}(\hat{A})$ defined as $\operatorname{Supp}(\hat{A})(x):=\operatorname{Supp}_{m}(\hat{A}(x))$ for any $x \in X$.

So, the hesitant fuzzy set support is simply built by taking the supports, in the crisp multiset sense, of the membership multiset $\hat{A}(x)$. We can now define an equivalence relation that accounts for repetition in much the same way as we did for the crisp multisets above.

Definition 4.5. Let $X$ be a universe and let $\hat{A}, \hat{B} \in \mathscr{F} \mathscr{M}(X)$ be two fuzzy multisets over $X . \hat{A}$ and $\hat{B}$ are said to be repetition-equivalent, $\hat{A} \sim_{r} \hat{B}$, if their hesitant fuzzy set supports are the same: $\operatorname{Supp}(\hat{A})=$ $\operatorname{Supp}(\hat{B})$.

The repetition equivalence relation makes it possible to define a bijection between its equivalence classes and the hesitant fuzzy sets over the universe.

Proposition 4.6. Given a universe $X$, there is a bijection $\phi_{M H}: \mathscr{F} \mathscr{M}(X) / \sim_{r} \longrightarrow \mathscr{F} \mathscr{H}(X)$ defined by

$$
\phi_{M H}([\hat{A}])=\operatorname{Supp}(\hat{A}) \quad[\hat{A}] \in \mathscr{F} \mathscr{M}(X) / \sim_{r}
$$

with $\hat{A}$ being any representative of the equivalence class $[\hat{A}]$. 
Proof. The function $\phi_{\mathrm{MH}}$ is defined for all equivalence classes $c \in \mathscr{F} \mathscr{M}(X) / \sim_{r}$. Given two fuzzy multisets $\hat{A}$ and $\hat{B}$, with equivalence classes $[\hat{A}]$ and $[\hat{B}]$ by $\sim_{r}$, their image by $\phi_{\mathrm{MH}}$ will be different if and only if $\operatorname{Supp}(\hat{A}) \neq \operatorname{Supp}(\hat{B})$, and then $[\hat{A}] \neq[\hat{B}]$, so it is injective.

On the other hand, given any hesitant fuzzy set $\tilde{A} \in \mathscr{F} \mathscr{H}(X)$, we can build a fuzzy multiset $\hat{A}$ defined for each $x \in X$ through a count function $\operatorname{Count}_{\hat{A}(x)}(t)=1$ if $t \in \tilde{A}(x)$ and $\operatorname{Count}_{\hat{A}(x)}(t)=0$ if $t \notin \tilde{A}(x)$ for $t \in[0,1]$. The hesitant fuzzy set support of $\hat{A}$ is obviously $\tilde{A}$, so $\phi_{\mathrm{MH}}([\hat{A}])=\tilde{A}$ for any hesitant fuzzy set $\tilde{A}$. Therefore, $\phi_{\mathrm{MH}}$ is surjective and then a bijection.

The existence of this bijection $\phi_{\mathrm{MH}}$ gives rigorous formal support to the intuitive identification between the hesitant fuzzy sets and the restricted fuzzy multisets where repetition is ignored (by considering the equivalence classes) or excluded (by considering the canonical representatives). But for this identification to be complete, we also need to prove that the common operations of complement, intersection and union commute with the bijection. And in order to show this, we first need to prove that the three operations are compatible with the repetition equivalence relation.

Proposition 4.7. Let $X$ be a universe. For any two fuzzy multisets over $X, \hat{A}, \hat{B} \in \mathscr{F} \mathscr{M}(X)$, if $\hat{A} \sim_{r} \hat{B}$ then $\hat{A}^{c} \sim_{r} \hat{B}^{c}$.

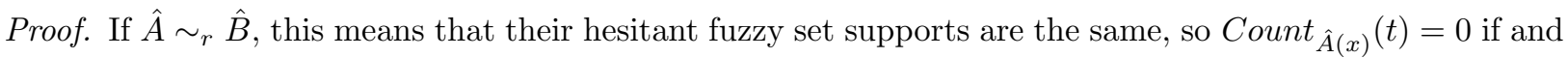

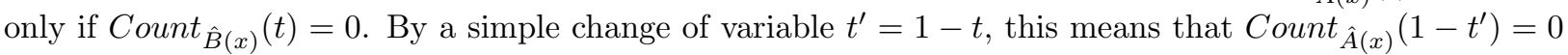
if and only if $\operatorname{Count}_{\hat{B}(x)}\left(1-t^{\prime}\right)=0$ and so $\operatorname{Supp}\left(\hat{A}^{c}\right)=\operatorname{Supp}\left(\hat{B}^{c}\right)$ and $\hat{A}^{c} \sim_{m} \hat{B}^{c}$.

This means that the equivalence classes are preserved by the complement operation and so the definition of complement can be brought forward to the quotient space $\mathscr{F} \mathscr{M}(X) / \sim_{m}$ by simply defining it in terms of any representative, as follows:

Definition 4.8. Let $X$ be a universe and let $[\hat{A}] \in \mathscr{F} \mathscr{M}(X) / \sim_{r}$ be a repetition equivalence class. The complement of $[\hat{A}]$ is the repetition equivalence class $[\hat{A}]^{c}$ defined by $[\hat{A}]^{c}:=\left[\hat{A}^{c}\right]$, with $\hat{A}^{c}$ being the complement, in the fuzzy multiset sense, of any representative $\hat{A}$ of the equivalence class $[\hat{A}]$.

In a similar way, we would expect the intersection and the union to be compatible with the repetition equivalence relation $\sim_{r}$ so that we can talk about intersections and unions of the equivalence classes in a meaningful way. It turns out, however, that among the definitions that we have provided for these operations, only the aggregate versions (definitions 3.4 and 3.5) that we propose in this article are compatible with $\sim_{r}$, a result which highlights the merits of the aggregate form.

Remark 4.9. Let's start by the intersection. As a very simple counterexample, consider a single-element universe $X=\{x\}$ and the three 3 -regular fuzzy multisets $\hat{A}_{1}(x)=\langle 0.1,0.1,0.3\rangle, \hat{A}_{2}(x)=\langle 0.1,0.3,0.3\rangle$ and $\hat{B}(x)=\langle 0.1,0.2,0.3\rangle$. And consider the ascending sort as the common sorting strategy: $s_{A}=s_{B}=s^{\uparrow}$. We have that $\hat{A}_{1} \sim_{r} \hat{A}_{2}$. But the 3-regular $s^{\uparrow}$-ordered intersection of $\hat{A}_{1}$ with $\hat{B}$ is $\hat{A}_{1} \cap_{s} \uparrow \hat{B}(x)=\langle 0.1,0.1,0.3\rangle$ whereas the intersection of $\hat{A}_{2}$ with $\hat{B}$ is $\hat{A}_{2} \cap_{s \uparrow} \hat{B}(x)=\langle 0.1,0.2,0.3\rangle$, so we have that $\hat{A}_{1} \sim_{r} \hat{A}_{2}$ but $\hat{A}_{1} \cap_{s \uparrow} \hat{B} \nsim_{r} \hat{A}_{2} \cap_{s \uparrow} \hat{B}$. Thus, the $m$-regular $\left(s_{A}, s_{B}\right)$-ordered intersection, as defined in Definition 3.1 , is not compatible with the repetition equivalence relation.

Similarly, under the conditions stated in their definitions, the $m$-regular $s$-ordered intersection (Definition 2.17), the regularised $s$-ordered intersection (Definition 2.22) and the regularised $\left(s_{A}, s_{B}\right)$-ordered intersection (Definition 3.3), being particular cases of the $m$-regular $\left(s_{A}, s_{B}\right)$-ordered intersection, are not compatible with the repetition equivalence relation either.

With regard to the union, if the consider the same counterexample, we have that he 3-regular $s^{\uparrow}$-ordered union of $\hat{A}_{1}$ with $\hat{B}$ is $\hat{A}_{1} \cup_{s \uparrow} \hat{B}(x)=\langle 0.1,0.2,0.3\rangle$ whereas the union of $\hat{A}_{2}$ with $\hat{B}$ is $\hat{A}_{2} \cup_{s \uparrow} \hat{B}(x)=$ $\langle 0.1,0.3,0.3\rangle$, so we have that $\hat{A}_{1} \sim_{r} \hat{A}_{2}$ but $\hat{A}_{1} \cup_{s \uparrow} \hat{B} \nsim_{r} \hat{A}_{2} \cap_{s \uparrow} \hat{B}$. Thus, the $m$-regular $\left(s_{A}, s_{B}\right)$-ordered union, as defined in Definition 3.2, is not compatible with the repetition equivalence relation and therefore, neither are the $m$-regular $s$-ordered union (Definition 2.18), the regularised $s$-ordered union (Definition 2.22) nor the regularised $\left(s_{A}, s_{B}\right)$-ordered union (Definition 3.3). 
Once we have presented counterexamples in the previous remark for any other considered definitions, we will now prove that definitions 3.5 and 3.6 are the only ones with a good behaviour.

Proposition 4.10. Let $X$ be a universe. The aggregate intersection $\cap^{a}$, as defined in Definition 3.4, is compatible with the repetition equivalence relation; i.e. given two pairs of fuzzy multisets over $X \hat{A}_{1}, \hat{B}_{1}, \hat{A}_{2}, \hat{B}_{2} \in$ $\mathscr{F} \mathscr{M}(X)$, if $\hat{A}_{1} \sim_{r} \hat{A}_{2}$ and $\hat{B}_{1} \sim_{r} \hat{B}_{2}$, then $\hat{A}_{1} \cap^{a} \hat{B}_{1} \sim_{r} \hat{A}_{2} \cap^{a} \hat{B}_{2}$.

Proof. By Definition 4.5, $\hat{A}_{1} \sim_{r} \hat{A}_{2}$ and $\hat{B}_{1} \sim_{r} \hat{B}_{2}$ means that their support hesitant fuzzy sets are equal, so we must prove that if $\operatorname{Supp}\left(\hat{A}_{1}\right)=\operatorname{Supp}\left(\hat{A}_{2}\right)$ and $\operatorname{Supp}\left(\hat{B}_{1}\right)=\operatorname{Supp}\left(\hat{B}_{2}\right)$ then $\operatorname{Supp}\left(\hat{A}_{1} \cap^{a} \hat{B}_{1}\right)=$ $\operatorname{Supp}\left(\hat{A}_{2} \cap^{a} \hat{B}_{2}\right)$. For each element of the universe $x \in X$, these are equalities between crisp sets. Let us take a value $t \in \operatorname{Supp}\left(\hat{A}_{1} \cap^{a} \hat{B}_{1}\right)(x)$. Because $t$ has ended up with a non-zero count value in the aggregate intersection, then it must have a non-zero count value in either of the original multisets $\hat{A}_{1}(x)$ or $\hat{B}_{1}(x)$. Without loss of generality, let us suppose that it is $t \in \operatorname{Supp}_{m}\left(\hat{A}_{1}(x)\right)$. Then, by repetition equivalence, $t \in \operatorname{Supp}_{m}\left(\hat{A}_{2}(x)\right)$ too. Now, the definition of the aggregate intersection as a multiset union of all the possible sequences made up of pairwise minima means that in order to have a non-zero count value in the intersection, $t \leq \min \left\{\max \left\{\operatorname{Supp}_{m}\left(\hat{A}_{1}(x)\right)\right\}, \max \left\{\operatorname{Supp}_{m}\left(\hat{B}_{1}(x)\right)\right\}\right\}$ and then, by the equality of the supports of $\hat{A}_{1}(x)$ and $\hat{A}_{2}(x)$ and $\hat{B}_{1}(x)$ and $\hat{B}_{2}(x)$, it follows that $t \leq \min \left\{\max \left\{\operatorname{Supp}_{m}\left(\hat{A}_{2}(x)\right)\right\}, \max \left\{\operatorname{Supp}_{m}\left(\hat{B}_{2}(x)\right)\right\}\right\}$ and since $t$ is in the support of $\hat{A}_{2}(x)$, then it will appear with a non-zero count value in the intersection $\hat{A}_{2} \cap^{a} \hat{B}_{2}(x)$, and so $t \in \operatorname{Supp}_{m}\left(\hat{A}_{2} \cap^{a} \hat{B}_{2}(x)\right)$. As the reasoning holds for any arbitrary value $t$ starting from either $\operatorname{Supp}_{m}\left(\hat{A}_{2} \cap^{a} \hat{B}_{2}(x)\right)$ or $\operatorname{Supp}_{m}\left(\hat{A}_{2} \cap^{a} \hat{B}_{2}(x)\right)$, the sets must be equal: $\operatorname{Supp}_{m}\left(\hat{A}_{2} \cap^{a} \hat{B}_{2}(x)\right)=$ $\operatorname{Supp}_{m}\left(\hat{A}_{2} \cap^{a} \hat{B}_{2}(x)\right)$, and this is for any $x \in X$, so $\operatorname{Supp}\left(\hat{A}_{2} \cap^{a} \hat{B}_{2}\right)=\operatorname{Supp}\left(\hat{A}_{2} \cap^{a} \hat{B}_{2}\right)$.

And the similar result for the aggregate union,

Proposition 4.11. Let $X$ be a universe. The aggregate union $\cup^{a}$, as defined in Definition 3.5, is compatible with the repetition equivalence relation; i.e. given two pairs of fuzzy multisets over $X \hat{A}_{1}, \hat{B}_{1}, \hat{A}_{2}, \hat{B}_{2} \in$ $\mathscr{F} \mathscr{M}(X)$, if $\hat{A}_{1} \sim_{r} \hat{A}_{2}$ and $\hat{B}_{1} \sim_{r} \hat{B}_{2}$, then $\hat{A}_{1} \cup \cup^{a} \hat{B}_{1} \sim_{r} \hat{A}_{2} \cup^{a} \hat{B}_{2}$.

Proof. The proof is virtually the same as the one for Proposition 4.10, substituting union for intersection and swapping the roles of the maxima and minima.

The fact that the equivalence classes are preserved by the aggregate intersection and union means that these operations can be adapted to the quotient space $\mathscr{F} \mathscr{H}(X) / \sim_{m}$ by simply defining them in terms of any representative, as follows:

Definition 4.12. Let $X$ be a universe and let $[\hat{A}],[\hat{B}] \in \mathscr{F} \mathscr{M}(X) / \sim_{r}$ be two repetition equivalence classes. The intersection of $[\hat{A}]$ and $[\hat{B}]$ is the repetition equivalence class $[\hat{A}] \cap[\hat{B}]$ defined by $[\hat{A}] \cap[\hat{B}]:=\left[\hat{A} \cap \cap^{a} \hat{B}\right]$, with $\hat{A} \cap^{a} \hat{B}$ being the aggregate intersection of any two representatives $\hat{A}$ and $\hat{B}$ of the equivalence classes $[\hat{A}]$ and $[\hat{B}]$.

Definition 4.13. Let $X$ be a universe and let $[\hat{A}],[\hat{B}] \in \mathscr{F} \mathscr{M}(X) / \sim_{r}$ be two repetition equivalence classes. The union of $[\hat{A}]$ and $[\hat{B}]$ is the repetition equivalence class $[\hat{A}] \cup[\hat{B}]$ defined by $[\hat{A}] \cup[\hat{B}]:=\left[\hat{A} \cup \cup^{a} \hat{B}\right]$, with $\hat{A} \cup^{a} \hat{B}$ being the aggregate union of any two representatives $\hat{A}$ and $\hat{B}$ of the equivalence classes $[\hat{A}]$ and $[\hat{B}]$.

Finally, we are now in a position to prove that these operations of complement, intersection and union commute with the bijection $\phi_{\mathrm{MH}}$.

Proposition 4.14. Let $X$ be a universe. The complement on $\mathscr{F} \mathscr{H}(X)$ and the complement on $\mathscr{F} \mathscr{M}(X) / \sim_{r}$ are related through the bijection $\phi_{M H}$ as follows

$$
\phi_{M H}\left([\hat{A}]^{c}\right)=\left(\phi_{M H}([\hat{A}])\right)^{c} \quad[\hat{A}] \in \mathscr{F} \mathscr{M}(X) / \sim_{r}
$$

Proof. This is an equality between hesitant fuzzy sets. First, by Definition $4.8,[\hat{A}]^{c}=\left[\hat{A}^{c}\right]$ and so the left-hand side can be rearranged as $\phi_{\mathrm{MH}}\left(\left[\hat{A}^{c}\right]\right)$, and, by the definition of $\phi_{\mathrm{MH}}$, (Proposition 4.6), this is the hesitant fuzzy set $\operatorname{Supp}\left(\hat{A}^{c}\right)$, where $\hat{A}^{c}$ is any representative of the equivalence class $\left[\hat{A}^{c}\right]$ and the complement 
of $\hat{A}$, a representative of the equivalence class $[\hat{A}]$. By definition of support, for any $x \in X, \operatorname{Supp}\left(\hat{A}^{c}\right)(x)=$ $\left\{t \in[0,1] \mid\right.$ Count $\left._{\hat{A}^{c}(x)}(t)>0\right\}$, which, by the definition of the hesitant fuzzy set complement (Definition 2.4), is the same as $\left\{t \in[0,1] \mid\right.$ Count $\left._{\hat{A}(x)}(1-t)>0\right\}$.

On the other hand, in the right-hand side we have to take the complement of the hesitant fuzzy set $\left(\phi_{\mathrm{MH}}([\hat{A}])\right)$, which is $\operatorname{Supp}(\hat{A})$ by the definition of $\phi_{\mathrm{MH}}$, where $\hat{A}$ is any representative of the equivalence class $[\hat{A}]$. And by definition of support, for any $x \in X, \operatorname{Supp}(\hat{A})(x)=\left\{t \in[0,1] \mid \operatorname{Count}_{\hat{A}(x)}(t)>0\right\}$ and by taking the hesitant fuzzy set complement, we get that the right-hand side of the equality evaluated at $x \in X$ is $\left\{1-t \in[0,1] \mid\right.$ Count $\left._{\hat{A}(x)}(t)>0\right\}$. And with a simple variable change $t^{\prime}=1-t$, this becomes $\left\{t^{\prime} \in[0,1] \mid\right.$ Count $\left._{\hat{A}(x)}\left(1-t^{\prime}\right)>0\right\}$, which is the same explicit expression that we reached for the left-hand side of the equality, thus proving the result.

Having established the result that the bijection $\phi_{\mathrm{MH}}$ commutes with the complement, as defined for the hesitant fuzzy sets and for the repetition equivalence classes of the fuzzy multisets, we will now show that it also commutes with the intersection and union.

Proposition 4.15. Let $X$ be a universe. The intersection on $\mathscr{F} \mathscr{H}(X)$ and the intersection on $\mathscr{F} \mathscr{M}(X) / \sim_{r}$ are related through the bijection $\phi_{M H}$ as follows

$$
\phi_{M H}([\hat{A}] \cap[\hat{B}])=\left(\phi_{M H}([\hat{A}])\right) \cap\left(\phi_{M H}([\hat{B}])\right) \quad[\hat{A}],[\hat{B}] \in \mathscr{F} \mathscr{M}(X) / \sim_{r} .
$$

Proof. First, by Definition 4.12, $[\hat{A}] \cap[\hat{B}]=\left[\hat{A} \cap^{a} \hat{B}\right]$, with $\hat{A}$ and $\hat{B}$ being two representatives of the equivalence classes $[\hat{A}]$ and $[\hat{B}]$, respectively. By the definition of $\phi_{\mathrm{MH}}$, (Proposition 4.6), the left-hand side of the equality is the hesitant fuzzy set $\operatorname{Supp}\left(\hat{A} \cap^{a} \hat{B}\right)$. And the support of the aggregate intersection, when evaluated at an $x \in X$, as we mentioned in the proof of Proposition 4.10, is the set $\{t \in \operatorname{Supp}(\hat{A})(x) \cup$ $\operatorname{Supp}(\hat{B})(x) \mid t \leq \min \{\max \{\operatorname{Supp}(\hat{A})(x)\}, \max \{\operatorname{Supp}(\hat{B})(x)\}\}$.

On the other hand, in the right-hand side we have to consider the two hesitant fuzzy sets $\left(\phi_{\mathrm{MH}}([\hat{A}])\right)$ and $\left(\phi_{\mathrm{MH}}([\hat{B}])\right)$, which are $\operatorname{Supp}(\hat{A})$ and $\operatorname{Supp}(\hat{B})$, respectively, by the definition of $\phi_{\mathrm{MH}}$, where $\hat{A}$ and $\hat{B}$ are any two representatives of the equivalence classes $[\hat{A}]$ and $[\hat{B}]$, respectively. And if we take the intersection of these two sets, in the standard hesitant fuzzy set sense of Definition 2.4, and evaluate it at $x \in X$, we get a set $\{t \in \operatorname{Supp}(\hat{A})(x) \cup \operatorname{Supp}(\hat{B})(x) \mid t \leq \min \{\max \{\operatorname{Supp}(\hat{A})(x)\}, \max \{\operatorname{Supp}(\hat{B})(x)\}\}\}$, which is the same explicit expression that we reached for the left-hand side of the equality, thus proving the result.

Proposition 4.16. Let $X$ be a universe. The union on $\mathscr{F} \mathscr{H}(X)$ and the union on $\mathscr{F} \mathscr{M}(X) / \sim_{r}$ are related through the bijection $\phi_{M H}$ as follows

$$
\phi_{M H}([\hat{A}] \cup[\hat{B}])=\left(\phi_{M H}([\hat{A}])\right) \cup\left(\phi_{M H}([\hat{B}])\right) \quad[\hat{A}],[\hat{B}] \in \mathscr{F} \mathscr{M}(X) / \sim_{r} .
$$

Proof. This can be proved in the same way as the intersection version of Proposition 4.15, substituting union for intersection and swapping the roles of the maxima and minima.

\section{Conclusion}

In this paper, we have studied in detail the main operations between fuzzy multisets, in order to obtain some coherent definitions from different points of view. We consider that fuzzy multisets are very appropriate to deal with real applications and this formal study can be useful in many context. Thus, although they are mathematically an extension of the typical hesitant fuzzy sets, their properties are much more interesting in some cases. However we have also investigated the close relationship that exists between the fuzzy multisets and the typical hesitant fuzzy sets, since this can be useful from the theoretical point of view. We have compared the definitions of the common operations of complement, intersection and union, and found some new definitions for the intersection and union of the fuzzy multisets, the aggregate intersection and union, that make it possible to identify the hesitant fuzzy sets with the subfamily of the fuzzy multisets where each membership value in $[0,1]$ has its multiplicity restricted to $\{0,1\}$. This confirms that the fundamental difference between the two theories is the way these binary operations are handled. 
We have seen the essential role played by ordered sequences, with the traditional fuzzy multiset definitions due to Miyamoto relying on arranging the values into sorted sequences, while the aggregate operations that match the hesitant theory take into account all the possible ordered sequences. This insight opens up new research possibilities, such as considering the ordered sequences as membership values themselves and studying the information loss in the experts' model due to the underlying order being ignored in fuzzy multisets and hesitant fuzzy sets.

Furthermore, we believe that the aggregate intersection and union can be used as the standard intersection and union for the multiset-based hesitant fuzzy sets, already mentioned in the original presentation of the hesitant fuzzy sets, but which have not received much attention as far as their basic operations and relations are concerned.

Finally, the relations among these the hesitant fuzzy sets and the fuzzy multisets together with the fuzzy sets can be summed up in the following diagram

\begin{tabular}{|c|c|c|c|c|}
\hline Fuzzy sets & $\mathscr{F}(X) \stackrel{\phi_{\mathrm{FH}}}{\longrightarrow}$ & $\mathscr{F} \mathscr{H}^{1}(X)$ & & \\
\hline Hesitant fuzzy sets & & $\mathscr{F} \mathscr{H}(X)$ & $\stackrel{\phi_{\mathrm{MH}}^{-1}}{\longrightarrow}$ & $\mathscr{F} \mathscr{M}(X) / \sim_{r}$ \\
\hline Fuzzy multisets & & & & $\mathscr{F} \mathscr{M}(X)$ \\
\hline
\end{tabular}

\section{Acknowledgments}

This work was partially supported by the Spanish Ministry of Science and Technology under projects TIN2014-59543-P and TIN-2017-87600-P.

\section{References}

[1] J.C.R. Alcantud, Some formal relationships among soft sets, fuzzy sets, and their extensions, International Journal of Approximate Reasoning 68 (2016) 45-53.

[2] J.C.R. Alcantud, V. Torra, Decomposition theorems and extension principles for hesitant fuzzy sets, Information Fusion $41(2018) 48-56$

[3] B. Bedregal, G. Beliakov, H. Bustince, T. Calvo, R. Mesiar, D. Paternain, A class of fuzzy multisets with a fixed number of memberships, Information Sciences 189 (2012) 1-17.

[4] L. Garmendia, R. González del Campo, J. Recasens, Partial orderings for hesitant fuzzy sets, International Journal of Approximate Reasoning 84 (2017) 159-167.

[5] I. Grattan-Guinness, Fuzzy Membership Mapped onto Intervals and Many-Valued Quantities, Mathematical Logic Quarterly 22 (1) (1976) 149-160.

[6] G.J. Klir, B. Yuan, Fuzzy Sets and Fuzzy Logic, Prentice Hall P T R, 1995.

[7] S. Miyamoto, Fuzzy Multisets and Their Generalizations, in: C.S. Calude, G. Păun, G. Rozenberg, A. Salomaa (Eds), Multiset Processing. WMC 2000. Lecture Notes in Computer Science, Springer, Berlin, Heidelberg, 2000, pp. 225-235.

[8] M. Mizumoto, K. Tanaka, Some Properties of Fuzzy Sets of Type 2, Information and Control, 31 (4) (1976) $312-340$.

[9] P. Quirós, P. Alonso, H. Bustince, I. Díaz, S. Montes, An entropy measure definition for finite interval-valued hesitant fuzzy sets, Knowledge-Based Systems 84 (2015) 121-133.

[10] A. Syropoulos, Mathematics of multisets, in: C.S. Calude, G. Păun, G. Rozenberg, A. Salomaa (Eds), Multiset Processing. WMC 2000. Lecture Notes in Computer Science, Springer, Berlin, Heidelberg, 2000, pp. 347-358.

[11] V. Torra, Hesitant Fuzzy Sets, International Journal of Intelligent Systems 25 (6) (2010) 529-539.

[12] M. Xia, Z. Xu, Hesitant fuzzy information aggregation in decision making, International Journal of Approximate Reasoning 52 (3) (2011) 395-407.

[13] Z. Xu, Hesitant Fuzzy Sets Theory, Springer, 2014.

[14] R.R. Yager, On the theory of Bags, International Journal of General Systems 13 (1) (1986) $23-37$.

[15] L.A. Zadeh, Fuzzy Sets, Information Control 8 (3 (1965) 338-353. 